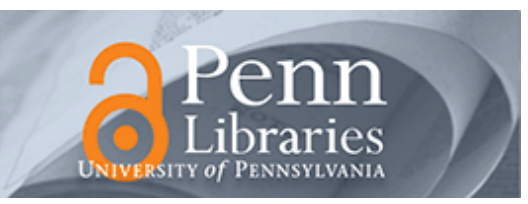

University of Pennsylvania

ScholarlyCommons

$12-9-2008$

\title{
Steering laws for distributed motion coordination of kinematic agents in three dimensions
}

\author{
Nima Moshtagh \\ University of Pennsylvania, nima@seas.upenn.edu \\ Ali Jadbabaie \\ University of Pennsylvania, jadbabai@seas.upenn.edu
}

Follow this and additional works at: https://repository.upenn.edu/ese_papers

\section{Recommended Citation}

Nima Moshtagh and Ali Jadbabaie, "Steering laws for distributed motion coordination of kinematic agents in three dimensions", . December 2008.

Moshtagh, N.; Jadbabaie, A., "Steering laws for distributed motion coordination of kinematic agents in three dimensions," Decision and Control, 2008. CDC 2008. 47th IEEE Conference on , vol., no., pp.1741-1746, 9-11 Dec. 2008 URL: http://ieeexplore.ieee.org/stamp/stamp.jsp?arnumber=4739155\&isnumber $=4738560$ Copyright 2008 IEEE. Reprinted from Proceedings of the 47th IEEE Conference on Decision and Control, 2008 (CDC 2008), vol., no., pp.1741-1746, 9-11 Dec. 2008.

This material is posted here with permission of the IEEE. Such permission of the IEEE does not in any way imply IEEE endorsement of any of the University of Pennsylvania's products or services. Internal or personal use of this material is permitted. However, permission to reprint/republish this material for advertising or promotional purposes or for creating new collective works for resale or redistribution must be obtained from the IEEE by writing to pubs-permissions@ieee.org. By choosing to view this document, you agree to all provisions of the copyright laws protecting it.

This paper is posted at ScholarlyCommons. https://repository.upenn.edu/ese_papers/480

For more information, please contact repository@pobox.upenn.edu. 


\title{
Steering laws for distributed motion coordination of kinematic agents in three dimensions
}

\author{
Abstract \\ We develop a systematic approach to synthesize distributed control laws for motion coordination in a \\ group of constant-speed kinematic rigid bodies. Parallel and balanced circular formations of mobile \\ agents moving in a three dimensional space are studied, and provably correct steering laws are \\ presented. The resulting steering laws have simple geometric intuitions which are based on the structure \\ of each particular formation.

\section{Comments} \\ Moshtagh, N.; Jadbabaie, A., "Steering laws for distributed motion coordination of kinematic agents in \\ three dimensions," Decision and Control, 2008. CDC 2008. 47th IEEE Conference on , vol., no., \\ pp.1741-1746, 9-11 Dec. 2008 URL: http://ieeexplore.ieee.org/stamp/ \\ stamp.jsp?arnumber $=4739155 \&$ isnumber $=4738560$ \\ Copyright 2008 IEEE. Reprinted from Proceedings of the 47th IEEE Conference on Decision and Control, \\ 2008 (CDC 2008), vol., no., pp.1741-1746, 9-11 Dec. 2008. \\ This material is posted here with permission of the IEEE. Such permission of the IEEE does not in any way \\ imply IEEE endorsement of any of the University of Pennsylvania's products or services. Internal or \\ personal use of this material is permitted. However, permission to reprint/republish this material for \\ advertising or promotional purposes or for creating new collective works for resale or redistribution must \\ be obtained from the IEEE by writing to pubs-permissions@ieee.org. By choosing to view this document, \\ you agree to all provisions of the copyright laws protecting it.
}




\title{
Steering Laws for Distributed Motion Coordination of Kinematic Agents in Three Dimensions
}

\author{
Nima Moshtagh ${ }^{\dagger}$, Ali Jadbabaie*
}

\begin{abstract}
We develop a systematic approach to synthesize distributed control laws for motion coordination in a group of constant-speed kinematic rigid bodies. Parallel and balanced circular formations of mobile agents moving in a three dimensional space are studied, and provably correct steering laws are presented. The resulting steering laws have simple geometric intuitions which are based on the structure of each particular formation.
\end{abstract}

\section{INTRODUCTION}

Different approaches to formation control can be categorized as leader-following [1], virtual structure [2], [3] and consensus approach [4]-[8]. The leader-following is usually implemented in a centralized fashion, whereas the consensus approach is decentralized. With the recent interest in distribute coordination in multi-agent systems, the consensus approach has received a lot of attention. We approach the formation control problem as a consensus problem [5], in the sense that robots communicate with their nearest neighbors so that they eventually reach an agreement in their motion, without the presence of a central commander.

Most of the research on distributed control of multiagent systems has been focused on planar systems [7][10]. Extension of the flocking results to three dimensions is nontrivial, because of higher degrees of freedom and a larger number of inputs for each robot. Some of the early works on controlling formation of rigid bodies in three-dimensions are [1], [11]. In [1] formation control for a team of nonholonomic aircraft was studied. By applying feedback linearization, the authors designed control laws for reaching a desired formation. In [11], Justh and Krishnaprasad represented the body frame of each agent by a natural Frenet frame that moved along the trajectory of each agent, and proposed a control law that stabilized the relative equilibria of rectilinear and circular formations. However, the they provided a proof of convergence only for the two-agent system. More recently, similar approach was undertaken in [12], [13] to develop motion coordination algorithms for distributed control of a group of nonholonomic rigid bodies in three dimensions.

\footnotetext{
* Ali Jadbabaie is with the GRASP Laboratory, University of Pennsylvania, Philadelphia, PA 19104. Ali Jadbabaie's research is supported in parts by the following grants: ARO-MURI W911NF-05-10381, ONR/YIP-542371, ONR N000140610436, and NSF-ECS-0347285. jadbabai@grasp. upenn. edu

Nima Moshtagh was with the GRASP Laboratory at University of Pennsylvania. He is currently a research engineer at Scientific Systems Company Inc., Woburn, MA 01803. nima.moshtaghessci.com
}

In our previous work [12] we presented preliminary results on motion coordination in a group of agents in three dimensions. We showed in [12] that the two seemingly different formation control laws that are given by Tanner et al. [14] and by Justh and Krishnaprasad [11] are actually the same laws, however the former is expressed in the inertial frame whereas the latter are expressed in the body coordinate system.

The main contribution of this paper is to present provably correct control laws for different types of coordinated behaviors such as parallel and circular formations. By appropriately defining the error terms we design control laws that minimize the total energy of the system and result in the convergence to a desired relative equilibria such a balanced circular formation. The resulting control laws depended only on the relative positions and orientations (i.e. the shape of the formation).

In Section III, we study the parallel formation of kinematic rigid bodies with constant speed. Each agent is controlled by a steering law that keeps the speed of each agent constant. The resulting cross-product steering law can be expressed in the body frame or the inertial frame depending on what coordinate system is used to express the parameters. Next, in Section IV, we study the generation of balanced circular formations on a sphere. Concluding remarks are given in Section V.

\section{BACKGRound AND PRoblem Statement}

\section{A. Rigid Body Motion}

Consider a group of $n$ unit-speed kinematic rigid bodies. Let the rotation matrix $R_{i}=\left[\begin{array}{lll}\mathbf{x}_{i} & \mathbf{y}_{i} & \mathbf{z}_{i}\end{array}\right] \in S O(3)$ represent the orientation of agent $i \in\{1, \ldots, n\}$ with respect to a fixed world frame, where $\mathbf{x}_{i}, \mathbf{y}_{i}$ and $\mathbf{z}_{i}$ are the columns of the rotation matrix. The kinematic equation describing the rotation of each agent is

$$
\dot{R}_{i}=\widehat{\boldsymbol{\omega}}_{i} R_{i},
$$

where $\boldsymbol{\omega}_{i}$ is the body angular velocity expressed in the (inertial) world frame, and $\widehat{\boldsymbol{\omega}}_{i}$ is the skew symmetric matrix of vector $\boldsymbol{\omega}_{i}$.

It is assumed that each kinematic agent moves with a constant unit-speed, i.e. $\left|\mathbf{v}_{i}\right|=1$, and its velocity vector is along the $\mathbf{z}_{i}$-axis of the body frame, therefore, $\mathbf{v}_{i}=R_{i} \mathbf{e}_{3}$ where $\mathbf{e}_{3}=\left[\begin{array}{lll}0 & 0 & 1\end{array}\right]^{T}$. Given $\mathbf{r}_{i}$ as the position vector of agent $i$, the kinematic equations describing the translational 
and rotational motion of agent $i$ are given by

$$
\begin{aligned}
\dot{\mathbf{r}}_{i} & =R_{i} \mathbf{e}_{3} \\
\dot{R}_{i} & =\widehat{\boldsymbol{\omega}}_{i} R_{i} .
\end{aligned}
$$

Since in this paper we are only concerned with the heading or the velocity vector of each agent, we can consider the reduced kinematics of each agent:

$$
\begin{aligned}
\dot{\mathbf{r}}_{i} & =\mathbf{v}_{i} \\
\dot{\mathbf{v}}_{i} & =\boldsymbol{\omega}_{i} \times \mathbf{v}_{i} .
\end{aligned}
$$

The control input of each agent is its angular velocity vector $\boldsymbol{\omega}_{i}$. Note that in this model, the acceleration vector $\dot{\mathbf{v}}_{i}$ (hence the force) is perpendicular to the velocity vector, thus, each rigid body is under a gyroscopic force [15] that only steers the agent without changing its speed.

\section{B. The Proximity Graph}

Each agent is capable of sensing information from its neighbors. The neighborhood set of agent $i, \mathcal{N}_{i}$, is a set of agents that can communicate with agent $i$. The size of the neighborhood depends on the characteristics of the sensors. We therefore assume that there is a pre-specified radius which determines the neighborhood relationship. The neighboring relationship between agents can be conveniently described by a proximity graph.

Definition 2.1: The proximity graph $\mathbb{G}=\{\mathcal{V}, \mathcal{E}, \mathcal{W}\}$ is a weighted graph consisting of:

- a set of vertices $\mathcal{V}$ indexed by the set of mobile agents;

- a set of edges $\mathcal{E}=\{(i, j) \mid i, i \in \mathcal{V}$, and $i \sim j\}$;

- a set of weights $\mathcal{W}$, over the set of edges $\mathcal{E}$.

If $i, j \in \mathcal{V}$, and $(i, j) \in \mathcal{E}$, then $i$ and $j$ are said to be adjacent, or neighbors and we denote this by writing $j \sim j$. If there is a path between any two vertices of a graph $\mathbb{G}$, then $\mathbb{G}$ is said to be connected. Given an orientation of the edges of a graph, we can define the incidence matrix of the graph to be a matrix $B$ with rows indexed by vertices and columns indexed by edges with entries of 1 representing the source of a directed edge and -1 representing the sink.

The Laplacian matrix $L(\mathbb{G})$ of graph $\mathbb{G}$ is represented in terms of its incidence matrix as $L=B B^{T}$ independent of the orientation of the edges. The Laplacian matrix captures many topological properties of the graph. The algebraic multiplicity of zero eigenvalue of the Laplacian $L$ (i.e. the dimension of its kernel) is equal to the number of connected components in the graph. The $n$-dimensional eigenvector associated with the zero eigenvalue is the vector of ones, $\mathbf{1}_{n}=[1, \ldots, 1]^{T}$. See [16] for more details on graph theory.

\section{Problem Statement}

Our goal in this paper is to design distributed control laws for each agent to generate swarm-like patterns such as parallel and circular motions. By distributed we mean that the agents interact and influence each other through the interaction among their controllers. Generally it is assumed that agents don't have access to global information, and only have limited communication abilities. Thus, the distributed control laws are based on local feedbacks.

Let us formally define the two types of coordinated motion in a multi-agent system:

Definition 2.2 (Parallel Formation): A group of mobile agents are in parallel formation when all the agents attain the same velocity vectors and distances between the agents are stabilized.

Definition 2.3 (Balanced Circular Formation): The set of equilibrium states where the agents are evenly spaced on circular trajectories, with a fixed geometric center, is called the balanced circular formation.

Note that in the above definitions, we do not care about the agreed upon direction of the velocity or center of the circle, just the fact that agreement has been reached.

\section{PARALlEL FORMATION}

We start with a parallel formation and synthesis a control law to achieve velocity alignment in a group of mobile agents in three dimensions. We have the following theorem:

Theorem 3.1: Consider $n$ agents with kinematics given by (3). If the proximity graph of the agents is fixed and connected, by applying input

$$
\boldsymbol{\omega}_{i}=\kappa \sum_{j \in \mathcal{N}_{i}}\left(\mathbf{v}_{i} \times \mathbf{v}_{j}\right), \quad \kappa>0
$$

the system converges to the set of relative equilibrium points given by $\boldsymbol{\omega}=\left[\begin{array}{lll}\boldsymbol{\omega}_{1}^{T} & \ldots & \boldsymbol{\omega}_{n}^{T}\end{array}\right]^{T}=\mathbf{0}$. Furthermore, the velocity consensus set is locally asymptotically attractive.

The control law (4) does not guarantee that the proximity graph of the multi-agent system remains connected, rather we claim that if graph $\mathbb{G}$ remains connected, then all the velocity vectors converge to the consensus set. The results are local, i.e. the region of convergence is not the entire sphere but rather it is only a hemisphere. In other words, if all the velocity vectors are initially in the same hemisphere, they converge to a common vector. The reason for this is that with the choice of input (4) the most we can claim is the invariance of a hemisphere resulting in convergence to a unique equilibrium. This point will be more clear in the proof of Theorem 3.1.

Proof: To show convergence to the equilibria we consider the following nonnegative function as a measure of the velocity misalignment:

$$
W(\mathbf{v})=\frac{1}{2} \sum_{i \sim j}\left|\mathbf{v}_{i}-\mathbf{v}_{j}\right|^{2}=\frac{1}{2} \mathbf{v}^{T} \bar{L} \mathbf{v}
$$

where $\mathbf{v}=\left[\mathbf{v}_{1}^{T}, \ldots, \mathbf{v}_{n}^{T}\right]^{T} \in \mathbb{R}^{3 n}$ is the stack vector of all the velocities and $\bar{L}=L \otimes I \in \mathbb{R}^{3 n \times 3 n}$, with $I$ being the $3 \times 3$ identity matrix, and $\otimes$ representing the Kronecher product. The time derivative of $W(\mathbf{v})$ along the trajectories of our 
system (3) is given by:

$$
\begin{aligned}
\dot{W}(\mathbf{v}) & =\dot{\mathbf{v}}^{T} \bar{L} \mathbf{v}=\sum_{i=1}^{n} \dot{\mathbf{v}}_{i}^{T}(\bar{L} \mathbf{v})_{i} \\
& =\sum_{i=1}^{n}\left(\boldsymbol{\omega}_{i} \times \mathbf{v}_{i}\right)^{T}(\bar{L} \mathbf{v})_{i} \\
& =-\sum_{i=1}^{n} \boldsymbol{\omega}_{i}^{T} \widehat{\mathbf{v}}_{i}^{T}(\bar{L} \mathbf{v})_{i} \leq 0
\end{aligned}
$$

where $\widehat{\mathbf{v}}_{i}$ is the skew-symmetric matrix of vector $\mathbf{v}_{i}$, and $(\bar{L} \mathbf{v})_{i} \in \mathbb{R}^{3}$ is a component of vector $\bar{L} \mathbf{v}$ that corresponds to the $i$-th agent. Noting that $\widehat{\mathbf{v}}_{i}$ is skew-symmetric, we have

$$
\begin{aligned}
\widehat{\mathbf{v}}_{i}^{T}(\bar{L} \mathbf{v})_{i} & =-\widehat{\mathbf{v}}_{i}(\bar{L} \mathbf{v})_{i}=-\mathbf{v}_{i} \times \sum_{j \in \mathcal{N}_{i}}\left(\mathbf{v}_{i}-\mathbf{v}_{j}\right) \\
& =\sum_{j \in \mathcal{N}_{i}}\left(\mathbf{v}_{i} \times \mathbf{v}_{j}\right)=\left(\frac{1}{\kappa}\right) \boldsymbol{\omega}_{i} .
\end{aligned}
$$

Thus, setting $\widehat{\mathbf{v}}_{i}^{T}(\bar{L} \mathbf{v})_{i}=(1 / \kappa) \boldsymbol{\omega}_{i}$ for a constant gain $\kappa>0$ guarantees that $W(\mathbf{v})$ decreases monotonically along trajectories of system (3):

$$
\dot{W}(\mathbf{v})=-\frac{1}{k} \sum_{i=1}^{n} \boldsymbol{\omega}_{i}^{T} \boldsymbol{\omega}_{i} \leq 0, \quad \kappa>0 .
$$

Consider the positively invariant set $\Omega_{c}=\{\mathbf{v} \mid W(\mathbf{v}) \leq$ $c \in \mathbb{R}\}$ characterized by the level-sets of $\mathrm{W}(\mathbf{v})$ over which $\dot{W} \leq 0 . \Omega_{c}$ is compact, because all the velocity vectors are unit-length, and thus, $W(\mathbf{v})$ is bounded. By LaSalle's invariance principle, every trajectory of the system starting in $\Omega_{c}$ converges to the largest invariant set contained in $\Gamma=\{\mathbf{v} \mid \dot{W}=0\}=\{\mathbf{v} \mid \boldsymbol{\omega}=\mathbf{0}\}$. The solution set of $\boldsymbol{\omega}=\mathbf{0}$ is given by the equilibria of

$$
\sum_{j \in \mathcal{N}_{i}}\left(\mathbf{v}_{i} \times \mathbf{v}_{j}\right)=\mathbf{0}, \quad \forall i \in\{1, \ldots, n\} .
$$

Note that $\Gamma$ is a very rich set and contains many equilibria other than the consensus set.

In order to show the local attractivity of the consensus set we first show that if all the velocity vectors start in the same hemisphere at time $t=0$, they will not leave that hemisphere, i.e. under control input (4) the initial hemisphere is positively invariant. We need to introduce the notion of geodesic versor $\mathbf{g}_{i}$. Let vector $\mathbf{g}_{i}$ be

$$
\mathbf{g}_{i}=\frac{\left(I-\mathbf{v}_{i} \mathbf{v}_{i}^{T}\right) \mathbf{v}_{i}^{\prime}}{\left|\left(I-\mathbf{v}_{i} \mathbf{v}_{i}^{T}\right) \mathbf{v}_{i}^{\prime}\right|}
$$

where $\mathbf{v}_{i}^{\prime}=\sum_{j \in \mathcal{N}_{i}} \mathbf{v}_{j}$ (See Figure 1). Thus, $\mathbf{g}_{i}$ is the unit vector along the projection of $\mathbf{v}_{i}^{\prime}$ perpendicular to $\mathbf{v}_{i}$. The control input (4), which is now equivalent to

$$
\boldsymbol{\omega}_{i}=\kappa\left(\mathbf{v}_{i} \times \mathbf{v}_{i}^{\prime}\right),
$$

can be interpreted as a geodesic control input [6] that rotates $\mathbf{v}_{i}$ along the geodesic versor $\mathbf{g}_{i}$ so that it is aligned with $\mathbf{v}_{i}^{\prime}$. Let $\alpha_{i}$ be the angle between $\mathbf{v}_{i}$ and $\mathbf{v}_{i}^{\prime}$. When $\alpha_{i} \in[0, \pi)$, i.e.

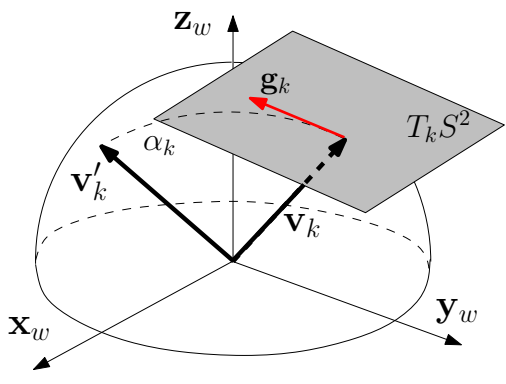

Fig. 1. The geodesic versor between $\mathbf{v}_{k}$ and $\mathbf{v}_{k}^{\prime}$ is defined as a unit vector perpendicular to $\mathbf{v}_{k}$ and pointing towards $\mathbf{v}_{k}^{\prime} . T_{k} S^{2}$ represents the tangent to the sphere $S^{2}$ at point corresponding to $\mathbf{v}_{k}$.

when all the initial velocity vectors start within a hemisphere, the geodesic versor will be unique.

Now, it is easy to see why the initial hemisphere is positively invariant under control law (4). Suppose $\mathbf{v}_{k}$ is the velocity vector that is about to leave the initial hemisphere. Since by the assumption all of its neighboring agents have velocity vectors inside the hemisphere, $\mathbf{v}_{k}^{\prime}$ is inside the hemisphere, hence the geodesic versor $\mathbf{g}_{k}$ points towards the inside of the hemisphere (see Figure 1). As a result $\mathbf{v}_{k}$ is pushed back towards the interior of the hemisphere. Thus, the initial hemisphere is positively invariant.

Once the invariance of the initial hemisphere is established, one can show that the synchronized state is the only stable equilibrium within the initial hemisphere. It was shown earlier that the equilibrium points are the set of solutions of $\boldsymbol{\omega}_{i}=\mathbf{0}, i \in\{1, \ldots, n\}$. Given that

$$
\begin{aligned}
\mathbf{v}_{i}^{\prime} & =\left(\mathbf{v}_{i}^{\prime T} \mathbf{v}_{i}\right) \mathbf{v}_{i}+\left(\mathbf{v}_{i}^{\prime T} \mathbf{g}_{i}\right) \mathbf{g}_{i} \\
& =\left|\mathbf{v}_{i}^{\prime}\right|\left(\cos \alpha_{i} \mathbf{v}_{i}+\sin \alpha_{i} \mathbf{g}_{i}\right)
\end{aligned}
$$

control input $\boldsymbol{\omega}_{i}$ can be written in terms of the angle $\alpha_{i}$ :

$$
\boldsymbol{\omega}_{i}=\kappa\left(\mathbf{v}_{i} \times \mathbf{v}_{i}^{\prime}\right)=\kappa\left|\mathbf{v}_{i}^{\prime}\right| \sin \alpha_{i}\left(\mathbf{v}_{i} \times \mathbf{g}_{i}\right) .
$$

Therefore, $\boldsymbol{\omega}_{i}=\mathbf{0}$ corresponds to $\left|\mathbf{v}_{i}^{\prime}\right|=0$ or $\sin \alpha_{i}=0$. Knowing the invariance of the interior of the initial hemisphere, and the assumption $\alpha_{i} \in[0, \pi)$, we conclude that the only solution corresponds to $\alpha_{i}=0$ for all $i \in\{1, \ldots, n\}$. Therefore, the velocity consensus set is locally attractive.

Figure 2 (a) shows the trajectories of five kinematic agents under the control law (4). Figure 2 (b) shows the initial and final orientations of the body frames of the same rigid bodies. The velocity vector of each agent is along its body $z$-axis, and as one can see at the final configuration, all the velocities are aligned, while the other axes are not.

Remark 3.2: Restricting the initial velocities to the interior of a hemisphere in three dimensions is the generalization of the local results given in [6] and [17] where all the headings were constrained to be within a half-circle characterized by $\theta \in(-\pi / 2, \pi / 2)$.

The advantage of the cross-product steering law (4) is that it can be easily computed in any desired coordinate system. When one is computing the control input for agent $i$, the 


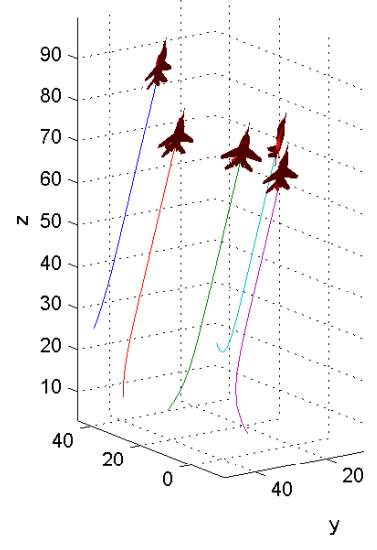

(a) Trajectories

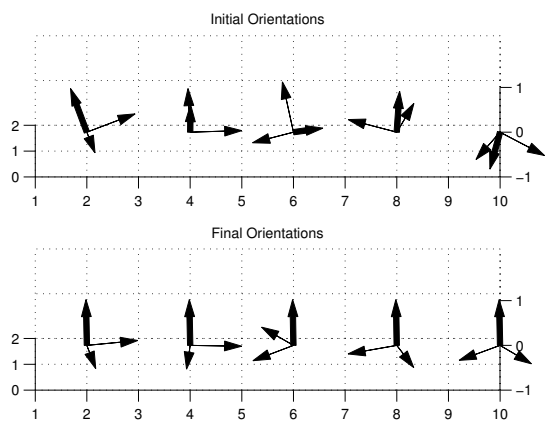

(b) Orientations

Fig. 2. (a) Trajectories of five rigid bodies applying the steering law (4) for velocity alignment. The underlying connectivity graph is fixed and connected. (b) The initial (top) and final (bottom) orientations of the same five rigid bodies with respect to a fixed inertial frame. The velocity of each agent is shown by a thicker arrow. At the equilibrium all the velocity vectors are aligned.

inputs become roll, $\omega_{i z}$, pitch, $\omega_{i x}$ and yaw, $\omega_{i y}$, which are the components of the body angular velocity expressed in the body frame. With all the velocity vectors expressed in the body frame ${ }^{i}\left(\mathbf{v}_{i}\right)=\left[\begin{array}{lll}0 & 0 & 1\end{array}\right]^{T}$, and ${ }^{i}\left(\mathbf{v}_{j}\right)=R_{i}^{T} \mathbf{v}_{j}=$ $\left[\left(\mathbf{x}_{i}^{T} \mathbf{v}_{j}\right)\left(\mathbf{y}_{i}^{T} \mathbf{v}_{j}\right)\left(\mathbf{z}_{i}^{T} \mathbf{v}_{j}\right)\right]^{T}$ we get

$$
\begin{aligned}
\omega_{i x} & =-\kappa \sum_{j \in \mathcal{N}_{i}} \mathbf{y}_{i}^{T} \mathbf{v}_{j}, \\
\omega_{i y} & =\kappa \sum_{j \in \mathcal{N}_{i}} \mathbf{x}_{i}^{T} \mathbf{v}_{j}, \\
\omega_{i z} & =0 .
\end{aligned}
$$

As it can be seen from (12) that roll, or rotation about the velocity axis, is zero. Note that (12) imposes a nonholonomic constraint on the motion of each rigid body in three dimensions.

\section{Circular Formations on Sphere}

Another important type of coordinated motion is a circular motion that is observed in nature [18], e.g. in the form of schooling of fish. The circular formation is a circular relative equilibrium in which all the particles travel around the same circle. The balanced formation is an interesting family of equilibrium states where the agents are evenly spaced on a circular trajectory, and their geometric center is fixed. Consider $n$ rigid bodies with kinematics

$$
\begin{aligned}
\dot{\mathbf{r}}_{i} & =\mathbf{z}_{i} \\
\dot{\mathbf{x}}_{i} & =\omega_{i z} \mathbf{y}_{i}-\omega_{i y} \mathbf{z}_{i} \\
\dot{\mathbf{y}}_{i} & =-\omega_{i z} \mathbf{x}_{i}+\omega_{i x} \mathbf{z}_{i} \\
\dot{\mathbf{z}}_{i} & =\omega_{i y} \mathbf{x}_{i}-\omega_{i x} \mathbf{y}_{i}, \quad i=1, \ldots, N
\end{aligned}
$$

which is equivalent to (2) but in terms of the body angular velocity $\boldsymbol{\omega}_{i}=\left[\begin{array}{lll}\omega_{i x} & \omega_{i y} & \omega_{i z}\end{array}\right]^{T}$ expressed in the body frame. The goal is to synthesis a control law for generating balanced circular formations about a circle with radius $\rho=1 / \omega_{o}$ where $\omega_{0}$ is a constant angular velocity term. The control law is designed by an appropriate choice of an error that is minimized at the desired relative equilibrium.

Let $\mathbf{c}_{i}$ represent the vector pointing towards the center of a circular trajectory of agent $i$. Given the assumption that the velocity vector is along the body $z$-axis, we have $\mathbf{c}_{i}=\mathbf{r}_{i}+\mathbf{d}_{i}$ where

$$
\mathbf{d}_{i}=\frac{1}{\omega_{o}}\left(\alpha \mathbf{x}_{i}+\beta \mathbf{y}_{i}\right), \quad \alpha, \beta \geq 0, \quad \alpha^{2}+\beta^{2}=1 .
$$

Figure 3 demonstrates the geometry of the circular trajectory under consideration.

Proposition 4.1: Consider a system of $n$ rigid bodies with kinematics (13). When the connectivity graph is a fixed and connected graph, by applying the control inputs:

$$
\begin{aligned}
& \omega_{i x}=-\beta \omega_{o}\left(1+<\mathbf{z}_{i}, \frac{(\bar{L} \mathbf{c})_{i}}{n}>\right) \\
& \omega_{i y}=\alpha \omega_{o}\left(1+<\mathbf{z}_{i}, \frac{(\bar{L} \mathbf{c})_{i}}{n}>\right) \\
& \omega_{i z}=-\alpha \omega_{o}<\mathbf{y}_{i}, \frac{(\bar{L} \mathbf{c})_{i}}{n}>+\beta \omega_{o}<\mathbf{x}_{i}, \frac{(\bar{L} \mathbf{c})_{i}}{n}
\end{aligned}
$$

where $\alpha^{2}+\beta^{2}=1$, the $n$-agent system asymptotically converges to circular trajectories on a sphere with radius $\rho_{o}=1 / \omega_{o}$

Proof: Consider the following function as a measure of the disagreement of the centers:

$$
S(\mathbf{c})=\frac{1}{2 n}<\mathbf{c}, \bar{L} \mathbf{c}>=\frac{1}{2 n} \sum_{i \sim j}\left|\mathbf{c}_{i}-\mathbf{c}_{j}\right|^{2} .
$$

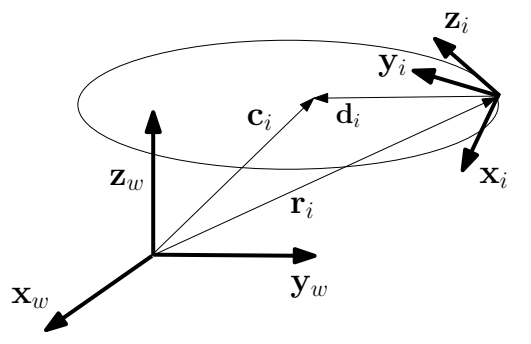

Fig. 3. The geometry of the circular trajectory. The velocity is along the body $z$-axis, and the center vector is defined by $\mathbf{c}_{i}=\mathbf{r}_{i}+\mathbf{d}_{i}$. 
Using the system kinematics (13), one can derive the dynamics of the center vector $\mathbf{c}_{i}$ :

$$
\dot{\mathbf{c}}_{i}=-\left(\beta \frac{\omega_{i z}}{\omega_{o}}\right) \mathbf{x}_{i}+\left(\alpha \frac{\omega_{i z}}{\omega_{o}}\right) \mathbf{y}_{i}+\left(\beta \frac{\omega_{i x}}{\omega_{o}}-\alpha \frac{\omega_{i y}}{\omega_{o}}+1\right) \mathbf{z}_{i}
$$

Now it is easy to show that using control inputs (14), (15) and (16) we have:

$$
\begin{aligned}
\dot{S}(\mathbf{c}) & =\frac{1}{n}<\dot{\mathbf{c}}, \bar{L} \mathbf{c}>=\sum_{i=1}^{n}<\dot{\mathbf{c}}_{i}, \frac{(\bar{L} \mathbf{c})_{i}}{n}> \\
& =\sum_{i=1}^{n}\left[-\left(1-\beta^{2}-\alpha^{2}\right)<\mathbf{z}_{i}, \frac{(\bar{L} \mathbf{c})_{i}}{n}>\right. \\
& -\left(\beta^{2}+\alpha^{2}\right)<\mathbf{z}_{i}, \frac{(\bar{L} \mathbf{c})_{i}}{n}>^{2} \\
& \left.-\left(\alpha<\mathbf{y}_{i}, \frac{(\bar{L} \mathbf{c})_{i}}{n}>-\beta<\mathbf{x}_{i}, \frac{(\bar{L} \mathbf{c})_{i}}{n}>\right)^{2}\right]
\end{aligned}
$$

If condition $\alpha^{2}+\beta^{2}=1$ holds, we get $\dot{S}(\mathbf{c}) \leq 0$. By an application of LaSalle's invariance principle, one can show that the trajectories of the system converge to largest invariant set defined by $\dot{S}(\mathbf{c})=0$. The corresponding equilibria are given by

$$
\begin{aligned}
\alpha<\mathbf{y}_{i}, \frac{(\bar{L} \mathbf{c})_{i}}{n}>-\beta & <\mathbf{x}_{i}, \frac{(\bar{L} \mathbf{c})_{i}}{n}>=0 \\
& <\mathbf{z}_{i}, \frac{(\bar{L} \mathbf{c})_{i}}{n}>=0
\end{aligned}
$$

Hence, at the equilibrium we have $\omega_{i x}=-\beta \omega_{o}, \omega_{i y}=\alpha \omega_{o}$ and $\omega_{i z}=0$, which correspond to a rotation about axis

$$
\boldsymbol{\omega}_{i}^{*}=\omega_{o}\left(-\beta \mathbf{x}_{i}+\alpha \mathbf{y}_{i}\right)
$$

Note that $\boldsymbol{\omega}_{i}^{*}$ is a vector perpendicular to $\mathbf{d}_{i}$ and $\left|\boldsymbol{\omega}_{i}^{*}\right|=\omega_{o}$, i.e. the norm of the angular velocity vector at the equilibrium is $\omega_{o}$. Thus, agents will traverse circular trajectories around a sphere with radius $\rho_{o}=1 / \omega_{o}$ and centered around $\mathbf{c}_{i}=$ $\mathbf{c}_{o} \in \mathbb{R}^{3}$, a fix point in space that depends on the initial positions of the rigid bodies.

Figure 4 (a) shows the trajectories of two agents under the above inputs. In order to make the agents to traverse the same great circles on a sphere, and also have balanced headings, we need to control the headings of the agents as well as the spacing of the circles. Let us consider the misalignment potential

$$
W(\mathbf{z})=\frac{1}{2 n}<\mathbf{z}, \bar{L} \mathbf{z}>=\frac{n}{2}-\frac{n}{2}|\mathbf{p}|^{2},
$$

where $\mathbf{p}=(1 / n) \sum_{i=1}^{n} \mathbf{z}_{i}$ is the average of the velocity vectors. $W(\mathbf{z})$ attains its minimum $(W(\mathbf{z})=0)$ at the synchronized state (all $\mathbf{z}_{i}$ 's are equal), and attains its maximum at the balanced state (all $\mathbf{z}_{i}$ 's are evenly spaced around a unit circle). Maximizing $W(\mathbf{z})$ corresponds to minimizing $U(\mathbf{z})=(n / 2)|\mathbf{p}|^{2}$.

Proposition 4.2: Consider a system of n kinematics rigid bodies described by (13). When the connectivity graph is a complete graph, by applying the angular velocity inputs

$$
\begin{aligned}
\omega_{i x} & =-\beta \omega_{o}-\omega_{o}\left(\beta<\mathbf{z}_{i}, \frac{(\bar{L} \mathbf{c})_{i}}{n}>-\omega_{o}<\mathbf{y}_{i}, \mathbf{p}>\right) \\
\omega_{i y} & =\alpha \omega_{o}+\omega_{o}\left(\alpha<\mathbf{z}_{i}, \frac{(\bar{L} \mathbf{c})_{i}}{n}>-\omega_{o}<\mathbf{x}_{i}, \mathbf{p}>\right) \\
\omega_{i z} & =-\alpha \omega_{o}<\mathbf{y}_{i}, \frac{(\bar{L} \mathbf{c})_{i}}{n}>+\beta \omega_{o}<\mathbf{x}_{i}, \frac{(\bar{L} \mathbf{c})_{i}}{n}>
\end{aligned}
$$

subject to the condition $\alpha^{2}+\beta^{2}=1$, the system converges to a balanced circular formation characterized by $\mathbf{p}=$ $(1 / n) \sum_{i=1}^{n} \mathbf{z}_{i}=\mathbf{0}$. All agents traverse great circles of radius $\rho_{o}=1 / \omega_{o}$.

Proof: Consider the composite potential function

$$
V(\mathbf{c}, \mathbf{z})=S(\mathbf{c})+U(\mathbf{z})=\frac{1}{2 n}<\mathbf{c}, \bar{L} \mathbf{c}>+\frac{n}{2}|\mathbf{p}|^{2} .
$$

Given (18) and the control inputs, the time derivative of $V(\mathbf{c}, \mathbf{z})$ along the trajectories of (13) is given by:

$$
\begin{aligned}
\dot{V} & =\sum_{i=1}^{n}\left[-\left(\frac{\omega_{i z}}{\omega_{o}}\right)^{2}+\left(1-\alpha^{2}-\beta^{2}\right)<\mathbf{z}_{i}, \frac{(\bar{L} \mathbf{c})_{i}}{n}>\right. \\
& +\omega_{o}<\left(\alpha \mathbf{x}_{i}+\beta \mathbf{y}_{i}\right), \mathbf{p}> \\
& -\left(\beta<\mathbf{z}_{i}, \frac{(\bar{L} \mathbf{c})_{i}}{n}>-\omega_{o}<\mathbf{y}_{i}, \mathbf{p}>\right)^{2} \\
& \left.-\left(\alpha<\mathbf{z}_{i}, \frac{(\bar{L} \mathbf{c})_{i}}{n}>-\omega_{o}<\mathbf{x}_{i}, \mathbf{p}>\right)^{2}\right] .
\end{aligned}
$$

Let $\mathbf{s}_{i}=\left(\alpha \mathbf{x}_{i}+\beta \mathbf{y}_{i}\right)$. Given that $\left(1-\alpha^{2}-\beta^{2}\right)=0$, and using the fact that near the balanced equilibrium $\sum_{i=1}^{n} \mathbf{s}_{i}$ equals to $\mathbf{p}^{\perp}$, we can show that $\dot{V}(\mathbf{c}, \mathbf{z}) \leq 0$. By an application of LaSalle's invariance principle, one shows that any trajectory of the system starting in the compact region $\Omega_{c}=\{(\mathbf{c}, \mathbf{z}) \mid \dot{V} \leq c\}$, converges to the largest invariant set within $\{(\mathbf{c}, \mathbf{z}) \mid \dot{V}(\mathbf{c}, \mathbf{z})=0\}$. This set is characterized by:

$$
\begin{aligned}
\alpha<\mathbf{y}_{i}, \frac{(\bar{L} \mathbf{c})_{i}}{n}>-\beta<\mathbf{x}_{i}, \frac{(\bar{L} \mathbf{c})_{i}}{n}> & =0 \\
\beta<\mathbf{z}_{i}, \frac{(\bar{L} \mathbf{c})_{i}}{n}>-\omega_{o}<\mathbf{y}_{i}, \mathbf{p}> & =0 . \\
\alpha<\mathbf{z}_{i}, \frac{(\bar{L} \mathbf{c})_{i}}{n}>-\omega_{o}<\mathbf{x}_{i}, \mathbf{p}> & =0 .
\end{aligned}
$$

Hence, at the equilibrium we have $\omega_{i x}=-\beta \omega_{o}, \omega_{i y}=\alpha \omega_{o}$ and $\omega_{i z}=0$, which correspond to a rotation about axis $\boldsymbol{\omega}_{i}^{*}=\omega_{o}\left[\begin{array}{lll}-\beta & \alpha & 0\end{array}\right]^{T}$, perpendicular to $\mathbf{d}_{i}=\left[\begin{array}{lll}\alpha & \beta & 0\end{array}\right]^{T}$.

From (19) we have that

$$
<\boldsymbol{\omega}_{i}^{*},(\bar{L} \mathbf{c})_{i}>=0, \quad i=1, \ldots, n .
$$

Since $\boldsymbol{\omega}_{i}^{*} \neq 0$, we conclude that $\bar{L} \mathbf{c}=\mathbf{0}$. Because $\mathbb{G}$ is a connected graph, we have $\mathbf{c} \in \operatorname{null}\{\bar{L}\}=\operatorname{span}\{\mathbf{1}\}$, i.e. $\mathbf{c}_{i}=\mathbf{c}_{o}$, where $\mathbf{c}_{o} \in \mathbb{R}^{3}$ is a fix point in space that depends on the initial positions of the rigid bodies. Also, since $\left|\boldsymbol{\omega}_{i}^{*}\right|=\omega_{o}$, we conclude that at the relative equilibrium all agents traverse circular trajectories around a sphere with radius $\rho_{o}=1 / \omega_{o}$ and centered around $\mathbf{c}_{o}$. 


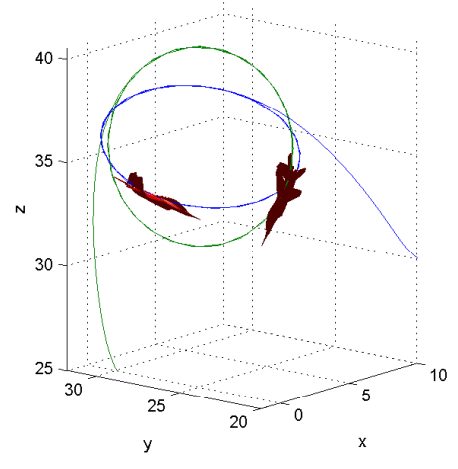

(a)

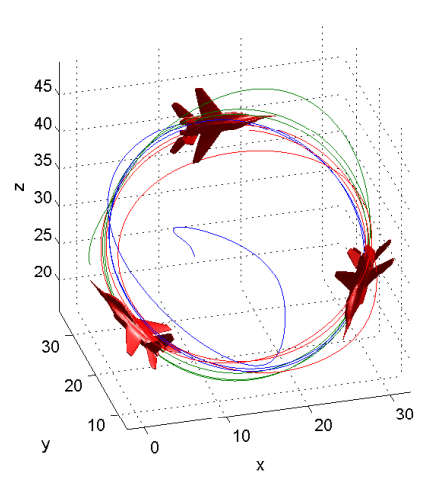

(b)

Fig. 4. Circular formations are achieved by minimizing the appropriate energy functions. (a) A circular motion on a sphere is generated by minimizing $S=(1 / 2 n)<\mathbf{c}, \bar{L} \mathbf{c}>$, (b) A balanced circular motion is generated by minimizing $V=S+U=(1 / 2 n)<\mathbf{c}, \bar{L} \mathbf{c}>+(n / 2)|\mathbf{p}|^{2}$.

From (20) and (21) we get $<\mathbf{x}_{i}, \mathbf{p}>=0$ and $<$ $\mathbf{y}_{i}, \mathbf{p}>=0, \forall i \in\{1, \ldots, n\}$. There are two possibilities: either $\mathbf{p}=\mathbf{0}$, which corresponds to a balanced formation, or $\mathbf{p} \perp \operatorname{span}\left\{\mathbf{x}_{i}, \mathbf{y}_{i}\right\}$ which corresponds to a configuration that $m$ agents are at antipodal position from the other $n-m$ agents $(1 \leq m<n / 2)$. The latter configurations are unstable. The only stable configurations are given by $\mathbf{p}=\mathbf{0}$. In three dimensions, only for $n=2,3$ one can guarantee that all agents travel the same circle. For $n>3$ the equilibria are great circles over which two or three agents are moving in a balanced formation.

See Figure 4 (b) for trajectories of three agents on a balanced circular trajectory.

\section{CONCLUSIONS}

In this work we presented a set of distributed control laws for motion coordination in a group of kinematic rigid bodies. We undertook a consensus approach to generate swarmslike behaviors such as parallel and circular formations. As a result, our approach is can be classified as a behavior-based method to formation control. The stability of the relative equilibria in each type of formation was studied in details.

The results on parallel motion of agents can be extended to scenarios where the underlying connectivity graph is statedependent (i.e. switch topologies). However, to achieve a balanced circular formation, the results hold only for an allto-all topology (complete graph) and a ring topology. We did not present the stability analysis of the ring-topology here, but the approach is similar to the one found in [19].

In future, we would like to extend our result to the control of the position and orientation of the circular trajectory that the agents traverse.

\section{REFERENCES}

[1] R. Fierro, C. Belta, J. Desai, and V. Kumar, "On controlling aircraft formations," in IEEE Conference on Decision and Control, vol. 2, 2001, pp. 1065-1070.

[2] W. Ren and R. W. Beard, "Decentralized scheme for spacecraft formation flying via the virtual structure approach," Journal of Guidance, Control, and Dynamics, vol. 27(1), pp. 73-82, January 2004.

[3] M. Mesbahi and F. Y. Hadaegh, "Formation flying of multiple spacecraft via graphs, matrix inequalities, and switching," in Proceedings of the IEEE International Conference on Control Applications, vol. 2, 1999 , pp. $1211-1216$

[4] A. Jadbabaie, J. Lin, and A. S. Morse, "Coordination of groups of mobile autonomous agents using nearest neighbor rules," IEEE Transactions on Automatic Control, vol. 48, no. 6, pp. 988-1001, June 2003.

[5] R. Olfati-Saber, "Flocking for multiagent dynamical systems: algorithms and theory," IEEE Transactions on Automatic Control, vol. 51, no. 3, pp. 401-420, March 2006.

[6] N. Moshtagh and A. Jadbabaie, "Distributed geodesic control laws for flocking of nonholonomic agents," IEEE Transaction on Automatic Control, vol. 52, pp. 681-686, april 2007.

[7] R. Sepulchre, D. Paley, and N. Leonard, "Collective motion and oscillator synchronization," in Cooperative Control, ser. Lecture Notes in Control and Information Science, V. Kumar, N. Leonard, and A. S. Morse, Eds. Springer, 2005, vol. 309, pp. 189-206.

[8] J. A. Fax and R. M. Murray, "Graph laplacians and stabilization of vehicle formations," 15th IFAC Congress, 2002.

[9] P. Ogren, E. Fiorelli, and N. Leonard, "Cooperative control of mobile sensing networks: Adaptive gradient climbing in a distributed environment," IEEE Transaction on Automatic Control, vol. 49(8), pp. 12921302, August 2004.

[10] Z. Lin, M. Brouke, and B. Francis, "Local control strategies for groups of mobile autonomous agents," IEEE Transactions on Automatic Control, vol. 49, no. 4, pp. 622-629, April 2004.

[11] E. Justh and P. Krishnaprasad, "Natural frames and interacting particles in three dimensions," in IEEE Conference on Decision and Control, December 2005, pp. $2841-2846$.

[12] N. Moshtagh, A. Jadbabaie, and K. Daniilidis, "Distributed coordination of dynamic rigid bodies," in IEEE Conference on Decision and Control, New Orleans, LA, December 2007, pp. 1480-1485.

[13] L. Scardovi, N. Leonard, , and R. Sepulchre, "Stabilization of collective motion in three dimensions: A consensus approach," in 46th IEEE Conference on Decision and Control, New Orleans, LA, Dec. 2007.

[14] H. Tanner, A. Jadbabaie, and G. Pappas, "Flocking in fixed and switching newtorks," IEEE Transaction of Automatic Control, vol. 52, pp. 863-868, 2007.

[15] E. Justh and P. Krishnaprasad, "Simple control laws for uav formation flying," Naval Research Laboratory Technical Report, June 2002.

[16] C. Godsil and G. Royle, Algebraic Graph Theory. New York: Springer Graduate Texts in Mathematics \# 207, 2001.

[17] A. Jadbabaie, N. Motee, and M. Barahona, "On the stability of Kuramoto model of coupled nonlinear oscillators," in Proceedings of the American Control Conference, vol. 5, July 2004, pp. 4296- 4301.

[18] I. Couzin, J. Krause, R. James, G. Ruxton, and N. Franks, "Collective memory and spatial sorting in animal groups," Journal of Theoretical Biology, vol. 218(1), p. 111, 2002.

[19] N. Moshtagh, N. Michael, A. Jadbabaie, and K. Daniilidis, "Visionbased, distributed control laws for motion coordination of nonholonomic robots," IEEE Transaction on Robotics, 2008, accepted. 